\title{
Phase coherence analysis of a field line resonance and solar wind oscillation
}

\author{
F. R. Fenrich ${ }^{1}$ and C. L. Waters ${ }^{2}$ \\ Received 22 July 2008; revised 8 September 2008; accepted 16 September 2008; published 17 October 2008.
}

[1] Discrete field line resonances (FLRs) are a common occurrence in the magnetosphere. However, the source of the stable, discrete FLR frequencies is still an unresolved problem. Recent work has proposed that discrete, continuous oscillations in the solar wind directly drive magnetospheric FLRs. However, these studies have only concentrated on common frequency and amplitude comparisons. This paper presents a new technique which utilizes either Fast Fourier Transform or wavelet crossphase measurements to demonstrate a high degree of phase coherence between a $1.7 \mathrm{mHz}$ FLR observed with the SuperDARN Kodiak radar and ACE SWE oscillations in solar wind plasma density. A statistical analysis which simulates the solar wind as red noise indicates with $99 \%$ confidence that the FLR oscillation is the same as the solar wind plasma density variation. Citation: Fenrich, F. R., and C. L. Waters (2008), Phase coherence analysis of a field line resonance and solar wind oscillation, Geophys. Res. Lett., 35, L20102, doi:10.1029/2008GL035430.

\section{Introduction}

[2] Field line resonances (FLRs) are standing MHD Alfven waves along geomagnetic field lines [Dungey, 1967; Radoski, 1967]. They are known to be associated with auroral arc formation [Lotko et al., 1998] and energization of radiation belt electrons [Rostoker et al., 1998; Degeling et al., 2008], both current topics of scientific study and debate. FLRs are also used for remotely sensing magnetospheric plasma densities [Waters et al., 2006]. Thus it is important to understand all aspects of this phenomenon. However, the source of the stable, monochromatic FLR frequencies remains an unresolved problem. Most often the discrete FLR frequencies are attributed to their coupling with magnetospheric cavity/waveguide modes [Kivelson and Southwood, 1985; Samson et al., 1992] or KelvinHelmholtz instabilities on the magnetospheric flanks [Southwood, 1974; Chen and Hasegawa, 1974]. However, these sources cannot reconcile the variable nature of the magnetosphere with the observed multiple discrete FLR frequencies [Fenrich et al., 1995].

[3] Recent work has proposed that discrete compressional MHD oscillations [Stephenson and Walker, 2002; Eriksson et al., 2006] and Alfvenic oscillations [Prikryl et $a l ., 1998]$ in the solar wind may directly drive magneto-

\footnotetext{
${ }^{1}$ Department of Physics, University of Alberta, Edmonton, Alberta, Canada.

${ }^{2}$ School of Mathematical and Physical Sciences, University of Newcastle, Newcastle, New South Wales, Australia.
}

Copyright 2008 by the American Geophysical Union. 0094-8276/08/2008GL035430\$05.00 spheric FLRs. Other studies have shown similarities between individual discrete solar wind compressional oscillations and global magnetospheric pulsations observed in geosynchronous magnetic fields [Kepko and Spence, 2003] and in low latitude ground based magnetometer measurements [Villante et al., 2007]. Kessel et al. [2004] reported that total Pc5 power of Alfvenic and compressional fluctuations in high speed solar wind streams was correlated with total Pc5 power of ground-based magnetic field pulsations. Furthermore, during individual high speed events they observed discrete frequency ULF fluctuations in the $1-4 \mathrm{mHz}$ band in the solar wind but no one-to-one correspondence with the ground-based frequencies. Except for Villante et al. [2007] who included some coherence spectra results, all of these studies have used frequency, amplitude, and direct time series comparisons. However, spectral cross-phase is an additional parameter which can be exploited. If solar wind oscillations drive FLRs then they should exhibit phase coherence with the FLR during the wave event. In this paper we present a new technique that utilizes either Fast Fourier Transform or wavelet crosspower and cross-phase measurements to determine the degree of phase coherence between a $1.7 \mathrm{mHz}$ FLR observed with the Super Dual Auroral Radar Network (SuperDARN) Kodiak HF radar [Greenwald et al., 1995] and oscillations in solar wind plasma density observed by the SWEPAM instrument on the ACE spacecraft [McComas et al., 1998]. A statistical analysis simulating the solar wind with red noise is included to determine the statistical significance of the cross-power and cross-phase results.

\section{Data Analysis}

[4] The FLR event under investigation occurred from 01:30 to 02:45 UT on 21 November, 2003. The magnetosphere was in the recovery phase of a large magnetic storm which began on 20 November, 2003. For the four hour interval surrounding the FLR event the ACE spacecraft measured above average magnetic fields, flow speed and density. The level 2, 64 second resolution, solar wind proton density data measured by the ACE SWEPAM instrument is shown with symbols in Figure 1a. A time lag of 65 min has been applied to the data based on the minimum variance method of Weimer et al. [2003]. The minimum variance method yielded a $\sim 50$ min delay to the location of the bowshock plus an additional 15 min was added to account for wave propagation through the magnetosheath and magnetosphere. Timing of the sharp increase in solar wind density at 01:30 UT to sharp positive deflections in GOES $10 \mathrm{~B}_{\mathrm{z}}$ and dayside 210 Magnetic Meridian magnetometer measurements yielded the same delay time. Note that delay times between $51 \mathrm{~min}$ to $80 \mathrm{~min}$ yield similar results. 


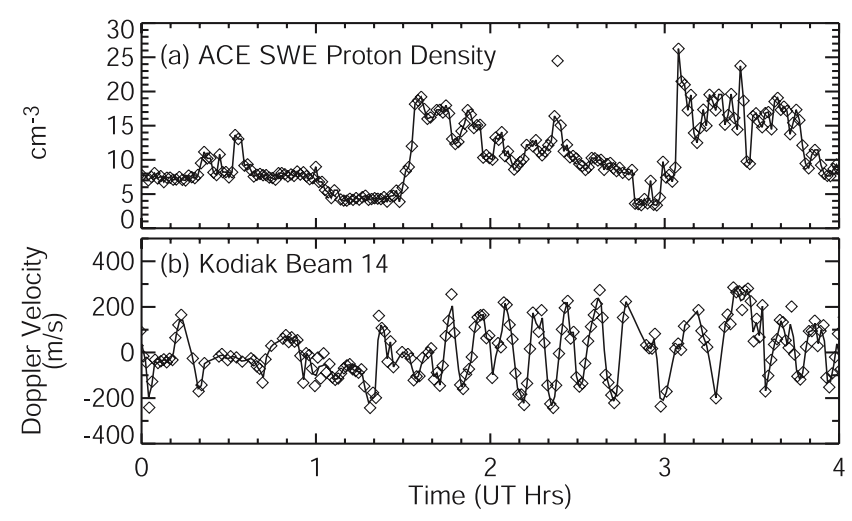

Figure 1. Raw data (symbols) and interpolated data (solid line) for (a) ACE SWE proton density and (b) SuperDARN Kodiak beam 14 Doppler velocity averaged over gates $9-$ 11 for the 00:00-04:00 UT interval on November 21, 2003. The ACE SWE data has been time lagged by $65 \mathrm{~min}$.

Following the sharp increase at 01:30 UT, the proton density clearly exhibits a $10 \mathrm{~min}$ period oscillation with peak to peak values of the order of $5 \mathrm{~cm}^{-3}$. One 'spike' in the data just after 02:20 UT has been removed. This point and 7 other single point gaps in the data were linearly interpolated and the resulting data are shown as the solid line in Figure 1a.

[5] Between 01:30 and 02:45 UT the SuperDARN Kodiak HF radar observed a $1.7 \mathrm{mHz}(10 \mathrm{~min}$ period) pulsation in the backscattered Doppler velocity. Each SuperDARN HF radar scans along 16 different azimuthal beam directions in succession with each beam spanning 3.2 degrees in azimuth while simultaneously sampling 75 range gates at $45 \mathrm{~km}$ resolution. The $1.7 \mathrm{mHz}$ pulsation observed by the Kodiak radar peaked in amplitude on range gates $9-11$ and was observed on all 16 radar beams. This pulsation was identified as a field line resonance with peak amplitude localized between $\sim 62-63$ degrees AACGM latitude and an azimuthal wave number of $\sim 4$. To facilitate cross-power and cross-phase analyses, the 60s sampled radar data from beam 14 were spatially averaged over range gates $9-11$ and then linearly interpolated to the same $64 \mathrm{~s}$ time points as the ACE data. The interpolated radar Doppler velocity data are shown as the solid line in Figure $1 b$. The symbols in Figure 1b indicate the 60s Doppler velocity data after spatial averaging.

[6] Before spectral analysis both data sets were detrended with a 30 min running average to remove low frequency trends. Dynamic Fast Fourier Transform (FFT) cross-power and cross-phase between the ACE SWE and Kodiak beam 14 data sets were calculated using a one hour FFT window with a Hanning weighting and a 1 point shift. Note that the cross-power spectrum of two time series is the discrete Fourier transform of their cross-correlation and the crossphase yields the phase difference between the two time series at each frequency [Ramirez, 1985]. If two data sets are correlated and exhibit a high degree of phase coherence at a specific frequency then the cross-phase measurement will be approximately constant with time. In order to provide a quantitative measure of the degree of phase coherence, the variance in cross-phase over one hour intervals at each frequency and time were calculated. The cross-power and variance in cross-phase between ACE SWE proton density and Kodiak Beam 14 data are shown in Figures $2 a$ and $2 b$, respectively. The lower values of the variance in cross-phase indicate coherent phase between the two time series.

[7] Given that random noise might generate low crossphase variance with the sliding FFT window method, we have used a red noise model to determine the statistical probability that the observed cross-power and phase coherence could be due to random noise in the solar wind. Note that red noise is characterized by a $1 / \mathrm{f}^{2}$ power law, similar to that of the solar wind [Goldstein and Roberts, 1999]. Thus 5000 four hour, red noise data sets were simulated for the solar wind using the equation $x_{\mathrm{i}}=x_{\mathrm{i}-1}+\operatorname{random}($ seed $) \times$ $\sigma \times 0.65$, where $\sigma$ is the standard deviation of the detrended four hour ACE SWE data set. The 0.65 multiplication factor was applied to match the standard deviations of the detrended red noise and solar wind data sets. The simulated red noise data sets were then cross-spectra analysed with the Kodiak Beam 14 radar data and the 5\% significant levels calculated at the times and frequencies when the radar data exhibited strong power. The 5\% significance levels correspond to the cross-power and variance in cross-phase values that will be reached or exceeded by simulated red noise data sets only $5 \%$ of the time. The black contour lines in Figures $2 \mathrm{a}$ and $2 \mathrm{~b}$ indicate these $5 \%$ significance levels with respect to red noise.

[8] For the FFT analysis used here a 1 hour FFT time interval has been used which limits the temporal resolution of the analysis. In order to improve this, we have repeated the processing using a wavelet analysis. The wavelet crosspower and cross-phase analysis between the ACE SWE and Kodiak Beam 14 data used the six hour interval from 23:00 UT on 20 Nov to $05: 00$ UT on 21 Nov using a Mortlet wavelet at $1 / 4$ octave frequency intervals [Waters et $a l ., 2006]$. The wavelet cross-power and variance in crossphase results for the 00:00-04:00 UT interval and 0.5$4 \mathrm{mHz}$ band are shown in Figures $3 \mathrm{a}$ and $3 \mathrm{~b}$. Note that in this wavelet transform the minimum frequency is $0.5 \mathrm{mHz}$ and the variance in cross-phase decreases with decreasing frequency unlike the FFT method. The solid black contour lines show the $5 \%$ significance level with respect to red noise.
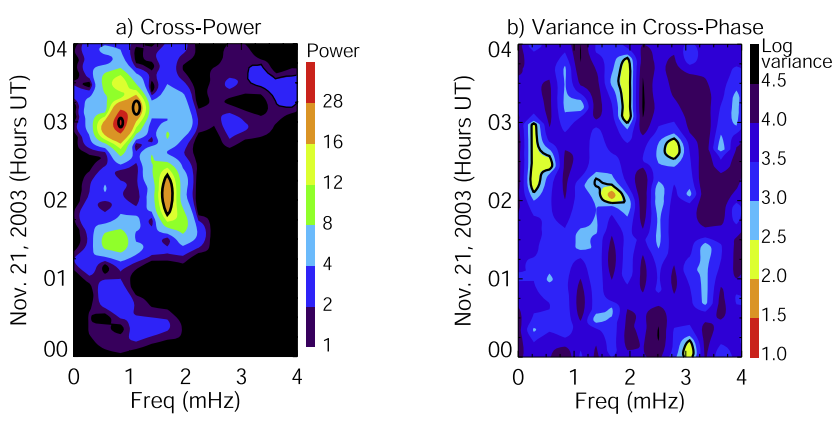

Figure 2. Plots of FFT (a) dynamic cross-power and (b) dynamic variance in cross-phase between ACE SWE density and Kodiak beam 14 Doppler velocity. The black contours correspond to the 5\% significant level with respect to red noise. 
[9] For comparison FFT cross-power and variance in cross-phase were calculated between Kodiak beam 14 and the other beams of the radar which also observed the $1.7 \mathrm{mHz}$ field line resonance. The cross-power and variance in cross-phase between beam 14 and beam 1 are shown in Figure 4 . The 5\% significant level shown in Figure 4 is calculated in the same manner as that described above for the solar wind density, however the standard deviation in this case corresponds to the four hour detrended Kodiak beam 1 data set. Figure 4 shows significant cross-power and variance in cross-phase values during the $1.7 \mathrm{mHz}$ FLR between $\sim 02: 00-02: 45$ UT. Similar values of significant cross power were seen between beam 14 and other beams of the radar during the $1.7 \mathrm{mHz}$ FLR. Significant values of variance in cross-phase were also seen between beam 14 and the other radar beams during the $1.7 \mathrm{mHz}$ FLR with the strongest levels of phase coherence found between beam 14 and adjacent beams for which minimum log of variance levels reached values of $\sim 1.0$.

\section{Discussion}

[10] Figure 1 clearly shows a $1.7 \mathrm{mHz}$ (10 min period) oscillation in ACE SWE density at the same time the $1.7 \mathrm{mHz}$ field line resonance is observed by the SuperDARN Kodiak radar between 01:30-02:45 UT. The FFT and wavelet cross-power and variance in cross-phase in Figures 2 and 3 show that the $1.7 \mathrm{mHz}$ FLR interval is the only interval that simultaneously exhibits both cross-power and variance in cross-phase above the $5 \%$ significance levels. Since power and phase are independent, the probability of this occurring randomly for a simulated red noise data set is only $1 \%$. Furthermore, the minimum level of variance in cross-phase with beam 14 during the $1.7 \mathrm{mHz}$ FLR is as low for the solar wind oscillation (Figure 2b) as it is for Kodiak beam 1 (Figure 4b). This indicates that the phase coherence between the solar wind plasma density oscillation and the $1.7 \mathrm{mHz}$ FLR is similar to the phase coherence between two extreme beams of the Kodiak radar.

[11] These results indicate that a high degree of phase coherence exists between the solar wind proton density oscillation and the $1.7 \mathrm{mHz}$ FLR. The observed values of phase coherence and cross-power are statistically unlikely to occur due to random chance. The $1.7 \mathrm{mHz}$ FLR pulsation begins at approximately the same time as the solar wind
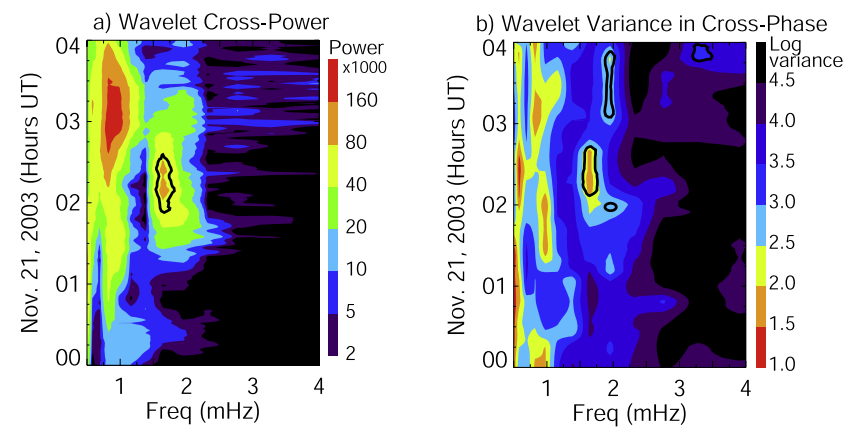

Figure 3. Wavelet (a) cross-power and (b) variance in cross-phase between ACE SWE density and Kodiak beam 14 Doppler velocity. The black contours correspond to the $5 \%$ significant level with respect to red noise.
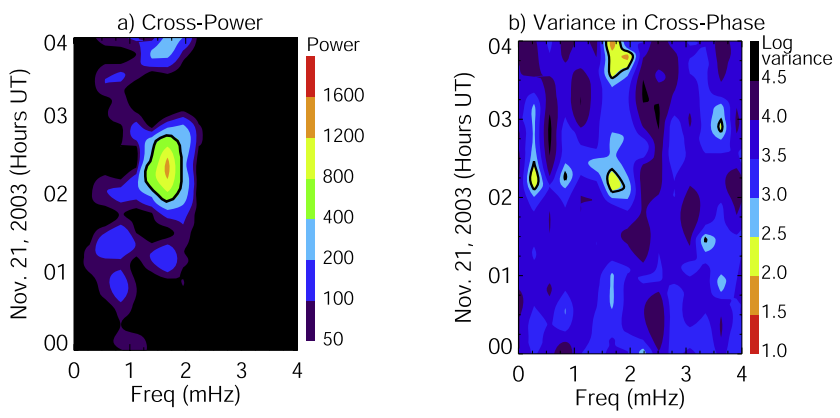

Figure 4. Dynamic plots of FFT (a) cross-power and (b) variance in cross-phase between Kodiak beam 1 and Kodiak beam 14 Doppler velocity. The black contours correspond to the $5 \%$ significant level with respect to red noise.

oscillation given an appropriate time delay and ceases approximately two cycles after the solar wind oscillation ends which suggests that the solar wind oscillation directly drives the FLR which decays away soon after the solar wind oscillation ceases.

[12] ACE total magnetic field measurements (not shown) also indicate a $1.7 \mathrm{mHz}$ oscillation that is coherent with the FLR and $180^{\circ}$ out of phase with the proton density oscillation suggesting this particular solar wind oscillation is a slow mode type compressional MHD wave. MHD waves are known to be modulated and amplified at the bowshock [Kessel et al., 2004] and can be transmitted through the magnetopause, preferentially when the magnetopause is open [Kwok and Lee, 1984]. For the 20-21 Nov, 2003 event the solar wind has a dominant IMF $\mathrm{B}_{\mathrm{y}}$ component making conditions favourable for magnetic reconnection on the flanks of the magnetopause. The $1.7 \mathrm{mHz}$ FLR observed by the Kodiak radar occurs at $~ 15: 30$ MLT and exhibits a westward or sunward phase propagation which is consistent with a source such as solar wind MHD compressional wave transmission through a dusk flank reconnection region. In addition, alignment of the solar wind compressional wave fronts along the spiral IMF direction can also result in westward phase propagation from dusk [Sibeck et al., 2003].

\section{Conclusions}

[13] A variance in cross-phase technique combined with cross-power and red noise statistics has been described. This method can identify FLRs that have similar discrete continuous oscillations in the solar wind with a high degree of confidence. For the 21 Nov, 2003 interval presented the analysis yields significant levels of cross-power and phase coherence between ACE SWE plasma density and Kodiak Doppler velocity data during a $1.7 \mathrm{mHz}$ FLR at 01:3002:45 UT suggesting that the FLR may be directly driven by the oscillation in the solar wind. For the $1.7 \mathrm{mHz}$ FLR event the degree of phase coherence is the same between two extreme beams of the radar as it is between the solar wind data and a single beam of the radar. In future more FLR events will be analyzed with this technique to determine the occurrence statistics of discrete FLRs associated with solar 
wind MHD waves, and to pinpoint any special conditions required for these events to occur.

[14] Acknowledgments. We thank D. J. McComas of Southwest Research Institute and the ACE SWEPAM team for providing the SWEPAM data. ACE Magnetic Fields Instrument data was provided by N. Ness of Bartol Research Institute and the MFI Team. We thank the SuperDARN principal investigators and the SuperDARN team for providing the SuperDARN radar data. We acknowledge $\mathrm{H}$. Singer of NOAA SEC and CDAWEB for use of the GOES 10 magnetometer data and K. Yumoto of Kyushu University for the 210 Magnetic Meridian chain data used in the solar wind time delay calculations. Financial support was provided by the Natural Sciences and Engineering Research Council of Canada.

\section{References}

Chen, L., and A. Hasegawa (1974), A theory of long-period magnetic pulsations: 1. Steady excitation of field line resonances, J. Geophys. Res., 79, 1024-1032.

Degeling, A. W., L. G. Ozeke, R. Rankin, I. R. Mann, and K. Kabin (2008), Drift resonant generation of peaked relativistic electron distributions by Pc 5 ULF waves, J. Geophys. Res., 113, A02208, doi:10.1029/ 2007JA012411.

Dungey, J. W. (1967), Hydromagnetic waves, in Physics of Geomagnetic Phenomena, vol. 2, edited by S. Matsushita and W. H. Campbell, pp. 913-934, Academic, San Diego, Calif.

Eriksson, P. T. I., A. D. M. Walker, and J. A. E. Stephenson (2006), A statistical correlation of Pc5 pulsations and solar wind pressure oscillations, Adv. Space Res., 38, 1763-1771.

Fenrich, F. R., J. C. Samson, G. Sofko, and R. A. Greenwald (1995), ULF high- and low-m field line resonances observed with the Super Dual Auroral Radar Network, J. Geophys. Res., 100, 21,535-21,547.

Goldstein, M. L., and D. A. Roberts (1999), Magnetohydrodynamic turbulence in the solar wind, Phys. Plasmas, 6, 4154.

Greenwald, R. A., et al. (1995), DARN/SUPERDARN A global view of the dynamics of high-latitude convection, Space Sci. Rev., 71, 761-796.

Kepko, L., and H. E. Spence (2003), Observations of discrete, global magnetospheric oscillations directly driven by solar wind density variations, J. Geophys. Res., 108(A6), 1257, doi:10.1029/2002JA009676.

Kessel, R. L., I. R. Mann, S. F. Fung, D. K. Milling, and N. O'Connell (2004), Correlation of Pc5 wave power inside and outside the magnetosphere during high speed streams, Ann. Geophys., 22, 629-641.

Kivelson, M. G., and D. J. Southwood (1985), Resonant ULF waves: A new interpretation, Geophys. Res. Lett., 12, 49-52.

Kwok, Y. C., and L. C. Lee (1984), Transmission of magnetohydrodynamic waves through the rotational discontinuity at the Earth's magnetopause, J. Geophys. Res., 89, 10,697-10,708.

Lotko, W., A. V. Streltsov, and C. W. Carlson (1998), Discrete auroral arc, electrostatic shock and suprathermal electrons powered by dispersive, anomalously resistive field line resonance, Geophys. Res. Lett., 25, $4449-4452$.

McComas, D. J., S. J. Bame, P. Barker, W. C. Feldman, J. L. Phillips, P. Riley, and J. W. Griffee (1998), Solar wind electron proton alpha monitor (SWEPAM) for the Advanced Composition Explorer, Space Sci. Rev., 86, 563-612.

Prikryl, P., R. A. Greenwald, G. J. Sofko, J. P. Villain, C. W. S. Ziesolleck, and E. Friis-Christensen (1998), Solar-wind-driven pulsed magnetic reconnection at the dayside magnetopause, Pc5 compressional oscillations, and field line resonances, J. Geophys. Res., 103, 17,307-17,322.

Radoski, H. R. (1967), Highly asymmetric MHD resonances: The guided poloidal mode, J. Geophys. Res., 72, 4026-4027.

Ramirez, R. W. (1985), The FFT, Fundamentals and Concepts, PrenticeHall, Englewood Cliffs, N.J.

Rostoker, G., S. Skone, and D. N. Baker (1998), On the origin of relativistic electrons in the magnetosphere associated with some geomagnetic storms, Geophys. Res. Lett., 25, 3701-3704.

Samson, J. C., B. G. Harold, J. M. Ruohoniemi, R. A. Greenwald, and A. D. M. Walker (1992), Field line resonances associated with MHD waveguides in the magnetosphere, Geophys. Res. Lett., 19, 441-444.

Sibeck, D. G., N. B. Trivedi, E. Zesta, R. B. Decker, H. J. Singer, A. Szabo, H. Tachihara, and J. Watermann (2003), Pressure-pulse interaction with the magnetosphere and ionosphere, J. Geophys. Res., 108(A2), 1095, doi:10.1029/2002JA009675.

Southwood, D. J. (1974), Some features of field line resonances in the magnetosphere, Planet. Space Sci., 22, 483-491.

Stephenson, J. A. E., and A. D. M. Walker (2002), HF radar observations of Pc5 ULF pulsations driven by the solar wind, Geophys. Res. Lett., 29(9), 1297, doi:10.1029/2001GL014291.

Villante, U., P. Francia, M. Vellante, P. Di Giuseppe, A. Nubile, and M. Piersanti (2007), Long-period oscillations at discrete frequencies: A comparative analysis of ground, magnetospheric, and interplanetary observations, J. Geophys. Res., 112, A04210, doi:10.1029/2006JA011896.

Waters, C. L., F. W. Menk, M. F. Thomsen, C. Foster, and F. R. Fenrich (2006), Remote sensing the magnetosphere using ground-based observations of ULF waves, in Magnetospheric ULF Waves: Synthesis and New Directions, Geophys. Monogr. Ser., vol. 169, edited by K. Takahashi et al., pp. 319-340, AGU, Washington, D.C.

Weimer, D. R., D. M. Ober, N. C. Maynard, M. R. Collier, D. J. McComas, N. F. Ness, C. W. Smith, and J. Watermann (2003), Predicting interplanetary magnetic field (IMF) propagation delay times using the minimum variance technique, J. Geophys. Res., 108(A1), 1026, doi:10.1029/ 2002JA009405.

F. R. Fenrich, Department of Physics, University of Alberta, Edmonton, AB T6G 2G7, Canada.

C. L. Waters, School of Mathematical and Physical Sciences, University of Newcastle, University Drive, Newcastle, NSW 2308, Australia. 